\title{
Spatial Effects on Hybrid Electric Vehicle Adoption
}

\author{
Xiaoli Liu ${ }^{\mathrm{a}}$, Matthew C. Roberts ${ }^{\mathrm{b}}$, Ramteen Sioshansi $\mathrm{i}^{\mathrm{a}, *}$ \\ ${ }^{a}$ Integrated Systems Engineering Department, The Ohio State University, 1971 Neil Avenue, Columbus, OH 43210, United \\ States of America \\ ${ }^{b}$ Agricultural, Environmental, and Development Economics Department, The Ohio State University, 2120 Fyffe Road, \\ Columbus, Ohio 43210, United States of America
}

\begin{abstract}
This paper examines spatial effects on hybrid-electric vehicle (HEV) adoption. This is in contrast to most existing analyses, which concentrate on analyzing socioeconomic factors and demographics. This paper uses a general spatial model to estimate the strength of 'neighbor effects' on HEV adoption-namely that each consumer's HEV-adoption decision can be influenced by the HEV-adoption decisions of geographic neighbors. We use detailed census tract-level demographic data from the 2010 United States Census and the 2012 American Community Survey and vehicle registration data collected by the Ohio Bureau of Motor Vehicles. We find that HEV adoption exhibits significant spatial effects. We further conduct a time-series analysis and show that historical HEV adoption has a spatial effect on future adoption. These results suggest that HEVs may appear in more dense clusters than models that do not consider spatial effects predict.
\end{abstract}

Keywords: Hybrid electric vehicle, technology adoption, spatial effects

\section{Introduction}

A hybrid-electric vehicle (HEV) contains an internal combustion engine and an electric propulsion system. This allows the vehicle's kinetic energy to be converted to electric energy that charges a battery when decelerating. This electric energy is used when the HEV subsequently accelerates to achieve better fuel economy. HEVs represent a rapidly developing transportation technology, which is widely accepted by customers compared to other types of non-conventional vehicles. Block and Harrison (2014) report that a total of about 3 million HEVs have been sold in the United States between 1999 and 2013. The Toyota Prius, which entered the United States market in the year 2000, led all HEV sales, representing a $41.8 \%$ market share (as of the end of the 2015 model year), followed by HEV versions of the Toyota Camry and Honda Civic. ${ }^{1}$

Predicting future HEV adoption is of interest for a number of reasons. Sioshansi and Denholm (2009, 2010) show that HEVs can introduce transportation energy and cost savings and associated emissions reductions relative to conventional vehicles. These efficiencies are of interest to policy makers. Another is that HEV adoption patterns can be used as a proxy for adoption of other more advanced transportation technologies that are not yet as mature as HEVs. This includes plug-in electric vehicles (PEVs). While HEV adoption is not a perfect proxy for PEV adoption, one can expect some similar adoption-dynamics between the two technologies. Egbue and Long (2012); Plötz et al. (2014) note that initial adoption of a new vehicle technology tends to be dominated by technology enthusiasts or early adopters. At the same time, Axsen and Kurani (2012) note interpersonal influences between early adopters of vehicle technologies and subsequent adopters (when the technology becomes more widely adopted). We believe that studying

\footnotetext{
* Corresponding author

Email addresses: liu.864@osu.edu (Xiaoli Liu), roberts.628@osu.edu (Matthew C. Roberts), sioshansi.1@osu.edu (Ramteen Sioshansi)

${ }^{1}$ http://www.afdc.energy.gov/data/
}

Preprint submitted to Transportation Research Part D: Transport and Environment

December 13, 2016 
such dynamics in the adoption of HEVs can provide useful insights into the possible future trajectory of PEV adoption. Finally, Tuttle and Baldick (2015) explicitly state that because of the advanced nature of HEV technology and adoption dynamics, a number of studies use past HEV adoption rates as a baseline from which to project future PEV adoption.

PEV adoption raises additional concerns relative to HEV adoption, because these vehicles include a grid-chargeable battery. The impacts of PEVs on the electric power system depends both on the rate at which they are adopted and the extent to which their owners are geographically clustered. As an example of this, Collins and Mader (1983); Mohseni and Stevie (2009) analyze possible PEV adoption in two regions of the United States. They find that significant clustering of PEVs is possible, which can yield extremely high distribution-level loads that may require significant infrastructure investments. Proactive planning of such investments requires knowing the extent to which PEVs may be clustered and where such clusters may appear.

Most empirical studies of HEV adoption focus on the effects of socioeconomic and demographic factors only. These studies reveal some geographic clustering of HEV adoption, because people with similar socioeconomics and demographics tend to cluster. Nevertheless, these studies do not capture the direct neighbor effect, which is that each consumer's HEV adoption decision can be influenced by HEV adoption decisions of geographic neighbors. Thus, these studies may result in biased estimates that underestimate geographic clustering.

In this paper we explicitly study such neighbor effects on HEV adoption, to fill this gap in the existing literature. We use several variants of a general spatial model to estimate the strength of these neighbor effects. Using demographic data from the 2010 United States Census and 2012 American Community Survey and vehicle registration data from the Ohio Bureau of Motor Vehicles, we demonstrate that HEV adoption exhibits statistically significant spatial effects. We also conduct a time-series analysis and show that historical HEV adoption has a significant spatial effect on future adoption. The remainder of this paper is organized as follows. Section 2 surveys other HEV adoption studies. Section 3 discusses the structure of the spatial models used in our analysis. We detail the data used in our analysis in Section 4 and summarize our results in Section 5. Section 6 concludes.

\section{Literature Review}

According to Musti and Kockelman (2011), unit vehicle price, vehicle type or class, and fuel economy are among the most important factors that affect vehicle-adoption decisions. Curtin et al. (2009) study the effects of economic considerations, environmental attitudes, and non-economic attitudes on HEV adoption. They conclude that although economic considerations have a significant influence on HEV purchase probabilities, environmental and other non-economic attitudes have an even larger impact. They also provide a model that can estimate HEV purchase probabilities of different households based on socioeconomic and demographic variables, including region (i.e., urban versus rural) of residence. Although region of residence is included in this model, it does not explicitly capture spatial effects. Despite this, their study suggests some clustering of HEV adoption due to the clustering of individuals with similar socioeconomics and demographics. Gallagher and Muehlegger (2011) examine the relative efficacy of different incentive mechanisms, such as tax-based subsidies, on consumer HEV adoption. As with the work of Curtin et al. (2009), this analysis neglects spatial effects. Rezvani et al. (2015) provide a comprehensive overview of the drivers for and barriers against consumer adoption of PEVs. Their work focuses on consumers' perceptions of PEVs, as this is an important driver of technology adoption.

Axsen and Kurani (2011) conduct one of the few analyses of PEV adoption to date that captures spatial impacts. Their study focuses on investigating the effects of social interactions on influencing perceptions of PEVs. This is done by mapping social networks, which gives a social episode diary, ranking the influence of different interpersonal experiences, and assessing how interpersonal interactions affect attitudes toward PEVs. To map people's social interactions, they conduct a detailed four- to six-week study of the social networks of individuals from 10 households. The work of Axsen and Kurani (2011) has two important distinctions compared to ours. First, because they focus on the spatial impacts of social networks on PEV adoption, their study relies on a small data set. This is because they must construct each subject's social 
network, which is a time-consuming process. Because our study focuses on the spatial impacts of geographic networks (i.e., the effects of HEV adoption by a spatial neighbor on an individual's adoption decision) we can use a much larger data set, which in our case covers the entire state of Ohio. This is, however, also a limitation of our analysis. We do not model the effects of HEV adoption by someone within an individual's social network that is not a geographic neighbor on vehicle-purchase decisions.

\section{Structure of Spatial Models}

Spatial analysis is successfully applied to many subfields of economics, including economic growth theory and regional and labor economics. According to Ward and Gleditsch (2008) the use of these techniques is motivated by interest in studying the interactions between social entities. This is because in many cases the outcomes of an individual's actions do not depend solely on that individual's attributes but also on the individual's physical location and interactions with others.

Anselin (1988); Paelinck and Klaassen (1979) describe spatial econometrics as a subfield of econometrics that deals with spatial interactions (i.e., autocorrelations) and structure (i.e., heterogeneity) in regression models for cross-sectional and panel data. LeSage and Pace (2009) identify two features that distinguish spatial from traditional econometric techniques: (i) spatial dependence exists between the observations or (ii) spatial heterogeneity occurs in the modeling relationships. Spatial econometrics is widely used in numerous research fields, including the social, political, and geographical sciences. This is because traditional econometric techniques assume homogeneity and no spatial correlation between observations, whereas spatial clustering is a common phenomenon in practice. Simply put, it is plausible to assume that observations are not independent of one another because most data are organized on a spatial template. Omitting spatial effects, if they exist, can result in inconsistent model estimates.

We begin in Section 3.1 by introducing the three spatial model types that we evaluate. We then discuss two standard methods to determine whether spatial effects may be important within a dataset in Section 3.2 and methods that can be used to determine which of the three models best capture the important spatial features in the data in Section 3.3. We finally discuss two interesting extensions of the basic spatial models in Sections 3.4 and 3.5, respectively - studying time-lagged and marginal effects.

\subsection{Spatial Econometric Models}

Anselin et al. (2004) survey a multitude of spatial econometric models. The three models that we choose to use here, the spatial autoregressive (SAR), spatial error (SE), and geographically weighted regression (GWR) models, are among the simplest spatial models. Moreover, they are conceptual analogs to nonspatial regression models and have proven themselves to be robust and flexible.

\subsubsection{Spatial Autoregressive Model}

The SAR model is used to capture a direct spillover effect from one spatial unit to another. This model has the form:

$$
y=\rho W y+X \beta+\epsilon,
$$

where $y$ is an $n \times 1$ vector of dependent variables, $X$ is an $n \times k$ matrix of explanatory variables, and $\epsilon$ is an $n \times 1$ vector of residuals. $\epsilon$ is assumed to have a mean-zero Gaussian distribution with variance-covariance matrix $\sigma^{2} I_{n}$, where $I_{n}$ is an $n \times n$ identity matrix. $W$ is the spatial-weighting matrix. The weighting matrix summarizes the relations between the spatial units, with $W_{i, j}$ reflecting the spatial influence of unit $j$ on $i$. The weighting matrix often has binary values, indicating whether two spatial units are spatially connected, for example whether two observations are contiguous. $W$ may also contain continuous values, for example, the distance between two observations. $\beta$ is a $k \times 1$ vector of coefficients and $\rho$ is a coefficient on the spatially autocorrelated dependent variable. Anselin (1988) provides a maximum-likelihood method for estimating the parameters of the SAR model. 


\subsubsection{Spatial Error Model}

The SE model is used to capture a spatial influence in the error terms only. The model has the form:

$$
y=X \beta+u,
$$

where:

$$
u=\lambda W u+\epsilon .
$$

The terms $y, X, \beta, W$, and $\epsilon$ have the same interpretation as in the SAR model. The parameter $\lambda$ is a coefficient on the spatially autocorrelated error term, $u$. Anselin (1988) also provides a maximum-likelihood method for estimating the SE model.

\subsubsection{Geographically Weighted Regression Model}

The GWR model is a generalization of the SAR and SE models, insomuch as it captures direct spillover effects from one spatial unit to another and spatial autocorrelation in the error terms. As such, the GWR model is the most general in that it can capture the types of autocorrelations that both the SAR and SE models can. The model has the form:

$$
y=\rho W_{1} y+X \beta+u,
$$

where:

$$
u=\lambda W_{2} u+\epsilon .
$$

The terms $y, \rho, X, \beta, u$, and $\epsilon$ have the same interpretation as in the SAR and SE models. $W_{1}$ and $W_{2}$ are spatial-weighting matrices on the autocorrelated dependent-variable and error terms, respectively. These two spatial-weighting matrices can, in general, differ, although one may choose to take $W_{1}=W_{2}$.

\subsection{Testing for Spatial Autocorrelation}

Spatial analysis is focused on two types of spatial relationships - autocorrelation within a dataset or autocorrelation in residuals. We outline two standard techniques used to test for these types of relationships.

\subsubsection{Moran's I: Testing for Autocorrelation Within a Dataset}

A standard method to measure spatial autocorrelation in a dataset is to use Moran's I. Moran (1950) defines the $I$ index as:

$$
I=\frac{N}{\sum_{i=1}^{N} \sum_{j=1}^{N} W_{i, j}} \cdot \frac{\sum_{i=1}^{N} \sum_{j=1}^{N} W_{i, j} \cdot\left(y_{i}-\bar{y}\right) \cdot\left(y_{j}-\bar{y}\right)}{\sum_{i=1}^{N}\left(y_{i}-\bar{y}\right)^{2}},
$$

where $N$ is the number of spatial units, which are indexed by $i$ and $j, y_{i}$ is the variable of interest and $\bar{y}$ its mean, and $W_{i, j}$ is the $(i, j)^{t h}$ element of the spatial-weighting matrix.

Under the null hypothesis of no spatial autocorrelation, the expected value of $I$ is:

$$
\mathbb{E}[I]=\frac{-1}{N-1},
$$

and its variance equals:

$$
\operatorname{Var}(I)=\frac{N^{2} W_{a}+N W_{b}+3 W_{0}^{2}}{W_{0}^{2} \cdot\left(N^{2}-1\right)}-\{\mathbb{E}[I]\}^{2},
$$

where:

$$
\begin{gathered}
W_{0}=\sum_{i=1}^{N} \sum_{j=1}^{N} W_{i, j}, \\
W_{a}=\frac{1}{2} \sum_{i=1}^{N} \sum_{j=1}^{N}\left(W_{i, j}+W_{j, i}\right)^{2},
\end{gathered}
$$


and:

$$
W_{b}=\sum_{i=1}^{N}\left(\sum_{j=1}^{N} W_{i, j}+\sum_{j=1}^{N} W_{j, i}\right)^{2} .
$$

Moran (1950) shows the derivations and interpretations of these terms. Using this mean and variance, Moran's $I$ can be converted to $Z$-scores:

$$
Z=\frac{I-\mathbb{E}[I]}{\sqrt{\operatorname{Var}(I)}},
$$

to test the hypothesis of no spatial autocorrelation.

\subsubsection{Lagrange Multiplier Error: Testing for Autocorrelation Within Errors}

The Lagrange multiplier error test is a common way to test for spatial autocorrelation in the residuals of a regression model. Spatially autocorrelated errors have the form:

$$
u=\lambda W u+\epsilon,
$$

where $\lambda$ is a coefficient on the spatially autocorrelated error, $W$ is the spatial-weighting matrix, and $\epsilon$ is a vector of mean-zero errors with variance-covariance matrix $\sigma^{2} I_{n}$, where $I_{n}$ is an $n \times n$ identity matrix. Under the null hypothesis that $\lambda=0$, meaning that there is no spatial autocorrelation in the errors, a standard regression model of the form:

$$
y=X \beta+\epsilon,
$$

can be estimated. The Lagrange multiplier error test statistic is defined as:

$$
L M_{e}=\frac{\left(\epsilon^{\top} W \epsilon / s^{2}\right)^{2}}{T},
$$

where:

$$
s^{2}=\frac{\epsilon^{\top} \epsilon}{N},
$$

and:

$$
T=\operatorname{tr}\left[\left(W+W^{\top}\right) W\right] .
$$

Under the null hypothesis of no spatial autocorrelation in the errors, $L M_{e}$ has a $\chi^{2}$ distribution with one degree of freedom.

\subsection{Selecting a Spatial Model}

Once it has been determined that spatial effects may be important within a dataset, the next question is what spatial model to use. It is important to note that estimating a model without spatial effects in such an instance can lead to biased and inconsistent parameter estimates. One possible method of selecting a spatial model, which LeSage and Pace (2009) mention, is to compare the log-likelihood value obtained after finding maximum-likelihood estimates for each model. This is because the log-likelihood function represents the likelihood that particular parameter values fit the observed data. The model with the greater log-likelihood value fits the observed data best among the models estimated.

If the log-likelihood values are not able to distinguish between the models (for instance, because the values are very close to one another), information criteria, such as Akaike Information Criterion (AIC) and Schwarz-Bayesian Information Criterion (SBC), can be used. AIC and BIC provide an estimate of the information lost when a given model is used to represent the process that generates a given dataset. It should be noted that AIC and SBC only provide information about the relative fit of two statistical models to a given dataset. They do not provide any absolute information. Thus, one cannot conclude whether a model is 'good' based on AIC or SBC. Rather, one can conclude that one model provides a better fit (based on information lost) than another. 


\subsection{Time-Lagged Spatial Model}

One potential use of a spatial econometric model is to understand the temporal dynamics of the spatial effects captured in the model. As discussed in Section 4, our case study takes the $y$ vector in these models to be the number of registered HEVs per 1000 passenger vehicles in each spatial unit. The $X$ matrix contains a number of demographic and socioeconomic variables for each spatial unit. Thus, the models allow us to conduct a static analysis, insomuch as we can study the direct and spatial relationships between HEV adoption and the demographic and socioeconomic characteristics of the spatial units at a certain point in time.

An important and interesting question may be to examine the temporal dynamics of HEV adoption. For example, one may want to know what effect time-lagged HEV adoption has on subsequent HEV adoption. A model with such a structure could allow one, for instance, to examine how HEV adoption by technology enthusiasts (during the early-adopter phase) affects subsequent adoption once the technology becomes more widely adopted.

Both the SAR and GWR models can be adapted to allow such a dynamic analysis. If we let $\dot{y}$ denote the time-lagged value of $y$, then the time-lagged SAR model has the form:

$$
y=\rho W y+\dot{\beta} W \dot{y}+X \beta+\epsilon,
$$

where $y, \rho, W, X, \beta$, and $\epsilon$ have the same interpretations and definitions as in the SAR model introduced in Section 3.1.1. The $W \dot{y}$ term captures the effect of historical values of $y$ on subsequent values of $y$. In our case, this would measure the impacts of historical HEV adoption on subsequent HEV adoption. The parameter, $\dot{\beta}$, is a scalar coefficient that measures the magnitude of this effect and is estimated along with $\rho$ and $\beta$ using the same maximum-likelihood method provided by Anselin (1988). Indeed, because $\dot{y}$ is not a dependent variable, it can be treated as an exogenous ' $X$ ' variable and this model can be estimated in the same manner as the standard SAR model.

The GWR model can be similarly generalized to include a time-lagged structure, which then takes the form:

$$
y=\rho W_{1} y+\dot{\beta} W_{1} \dot{y}+X \beta+u,
$$

where $y, \rho, W_{1}, X, \beta$, and $u$ have the same definitions as in the standard GWR model introduced in Section 3.1.3 and $\dot{\beta}$ and $\dot{y}$ have the same interpretations as in the time-lagged SAR model. Again, the $W_{1} \dot{y}$ term has the same interpretation as in the time-lagged SAR model as capturing the effect of historical values of $y$ on subsequent values of $y$. Moreover, because $\dot{y}$ is not a dependent variable, it can be treated as an exogenous ' $X$ ' variable and this model can be estimated in the same manner as the standard GWR model.

The SE model can also be generalized to include a time-lagged structure. The time-lagged form of the SE model takes the form:

$$
y=\dot{\beta} W_{1} \dot{y}+X \beta+u,
$$

where $y, W_{1}, X, \beta$, and $u$ have the same definitions as in the standard SE model introduced in Section 3.1.2 and $\dot{\beta}$ and $\dot{y}$ have the same interpretations as in the time-lagged SAR and GWR models. Because $\dot{y}$ is not a dependent variable, this model can be estimated using the same maximum-likelihood method suggested by Anselin (1988) for estimating the standard SE model.

\subsection{Marginal Effect Analysis}

A natural question that the use of a spatial econometric model raises is how to determine the marginal effect of the dependent variable in one spatial unit on dependent-variable values in other spatial units. To demonstrate how such an analysis is done, consider the standard GWR model:

$$
y=\rho W_{1} y+X \beta+\lambda W_{2} u+\epsilon,
$$

where all of the variables and parameters have the same interpretation as in Section 3.1.3. 
Let $\hat{\rho}, \hat{\beta}$, and $\hat{\lambda}$ be maximum-likelihood coefficient estimates of the GWR model. Suppose that we are interested in examining what effect a change in the value of $y$ for one spatial unit has on the values of $y$ for other spatial units. More specifically, suppose that:

$$
y^{0}=\left(\begin{array}{c}
y_{1}^{0} \\
\vdots \\
y_{n}^{0}
\end{array}\right),
$$

denotes the starting values of the dependent variable. We would like to examine the effect of a change in the value of $y$ in spatial unit $i$ on the values of $y$ in other spatial units. To do so, define:

$$
y^{\Delta}=\left(\begin{array}{c}
y_{1}^{0} \\
\vdots \\
y_{i-1}^{0} \\
y_{i}^{0}+\Delta_{i} \\
y_{i+1}^{0} \\
\vdots \\
y_{n}^{0}
\end{array}\right),
$$

as the perturbed dependent-variable vector, where $\Delta_{i}$ is the change in $y$ in spatial unit $i$. One can estimate the expected value of $y$, which we denote $y^{1}$, by computing:

$$
y^{1}=\hat{\rho} W_{1} y^{\Delta}+X \hat{\beta}+\hat{\lambda} W_{2} u .
$$

The difference, $\left(y^{1}-y^{0}\right)$, provides an estimate of the partial marginal effect of $y$ in spatial unit $i$ on the values of $y$ in other spatial units. The reason that $\left(y^{1}-y^{0}\right)$ provides an estimate of the partial marginal effect is that it only accounts for the effect of $y$ in spatial unit $i$ on other spatial units. Changes in the values of $y$ in other spatial units will, in turn, have a spillover effect on $y$ in spatial unit $i$. This spillover effect is not captured by $y^{1}$.

To estimate the full marginal effect of $y$ in spatial unit $i$ on the values of $y$ in other spatial units, one must use an iterative estimation process. Let $y^{k}$ denote the estimate of the expected value of $y$ after $k$ iterations. The $y^{k}$ 's are computed iteratively as:

$$
y^{1}=\hat{\rho} W_{1} y^{\Delta}+X \hat{\beta}+\hat{\lambda} W_{2} u,
$$

and:

$$
y^{k+1}=\hat{\rho} W_{1} y^{k}+X \hat{\beta}+\hat{\lambda} W_{2} u, \forall k \geq 1 .
$$

Successive values of the $y^{k}$ 's are computed using (26) until we have that $y^{k}=y^{k-1}$, meaning that $y^{k}$ captures all of the spatial spillover effects. This type of a marginal analysis provides a more concrete interpretation of the dependent variable and coefficient estimates in the SAR and GWR models.

\section{Case Study Data}

Our case study focuses on HEV adoption in the state of Ohio. We use two main data sets, which are discussed in this section, for our analysis. In Section 4.1 we discuss the vehicle registration data, which are gathered from the Ohio Bureau of Motor Vehicles (BMV). Section 4.2 introduces the census-tract-level socioeconomic and demographic data used, which are obtained from the United States Census Bureau. Finally, Section 4.3 explains how the spatial weighting matrices in the SAR, SE, and GWR models are determined and some limitations of our spatial modeling. 


\subsection{Ohio Bureau of Motor Vehicles Data}

The BMV reports data for every motor vehicle registered in the state of Ohio. This includes vehicle identification number (VIN), registration date, registration lapse date (if applicable), and registration address. Vehicles are also categorized by type, for example passenger vehicles (including light-duty trucks), heavy-duty trucks, buses, and municipal- and government-owned vehicles. Because our analysis is focused on HEV adoption by individuals, we only consider passenger vehicles in our analysis.

We study the adoption penetration of HEVs within spatial units across the state of Ohio. We use the 2943 census tracts within Ohio, defined by the United States Census Bureau, as the spatial units in our analysis. Census tracts are small and relatively permanent statistical subdivisions of the state. Census tracts are, however, updated prior to each decennial census as part of the United States Census Bureau's Participant Statistical Areas Program. The primary purpose of census tracts is to provide a stable set of geographic units for the presentation of statistical data. Census tracts have a target size of 4000 people, though in practice, they can range between 1200 and 8000. Unlike other geographic subdivisions (e.g., postal codes) census tracts remain relatively stable over each decade. We define the adoption penetration of HEVs within each census tract as the number of HEVs registered per 1000 passenger vehicles (including HEVs and other vehicle types) registered between the years 2001 and 2012. These adoption penetrations are the 'left-hand-side' or ' $y$ ' variables in our spatial models. Our analysis uses vehicle registrations that are unlapsed as of the end of 2012 - a lapsed registration typically indicates that a vehicle has been resold, taken out of state, or is otherwise not in regular use within the state of Ohio by the original registrant. A vehicle that is resold within the state of Ohio appears in the BMV dataset as a lapsed registration by the original owner and a new registration by the new owner. Thus, we use the most recent unlapsed registration for any VINs that appear in the BMV data multiple times.

Vehicle registrations are mapped to census blocks using the registration address reported in the BMV data and Esri's ArcGIS software. We have an $85 \%$ geocoding match rate, i.e., $15 \%$ of vehicle registrations are not successfully associated with a census block and are excluded from our analysis. Unmatched vehicle registrations typically represent a data-quality issue. One common data-quality issue is poor data entry by BMV employees or others entering vehicle-registration data into the BMV database. Excluding vehicle registrations that have unmatched addresses could bias our model estimates if there is a systematic bias in which vehicles have unmatched addresses (e.g., vehicles from certain census tracts or certain types of vehicles). Our analysis of the unmatched vehicle registrations does not show any such biases. Thus, we do not expect any important biases being introduced to our model estimates as a result.

We identify which of the vehicles registered in each census block are HEVs based on VINs. Specifically, each vehicle manufacturer encodes vehicle model, powertrain, body type, and other information into the VIN. This encoding is not standardized among vehicle manufacturers, however. Moreover, VIN encoding can change from one generation of a vehicle model to the next. We are able to find regular expressions to decode VINs for the eight highest-selling HEV models, which represent about $85 \%$ of HEV sales in the United States. As with the unmatched vehicle registrations, excluding HEVs for which we are not able to decode VINs could bias our model estimates if there is a systematic bias in where certain HEV makes or models are registered. While we do believe that there is spatial clustering of HEVs within the state, we do not believe that there is strong clustering of different HEV makes or models. Table 1 summarizes the number of each HEV type that is newly registered in the state in each of the years in the study period and total registrations over the entire study period. It also provides each year's and the overall HEV adoption penetration for the entire study period.

\subsection{United States Census Bureau Data}

The United States Census Bureau reports demographic and socioeconomic data collected in the 2010 decennial United States Census and the 2012 American Communities Survey (ACS), which are used in our analysis. We collect census tract-level data on income, age, education, population size, commuting patterns, and vehicle ownership for residents of each census tract. Although the census and ACS include data for many pertinent variables, our analysis considers the variables listed in Table 2 only, as these provide reasonable model fits without introducing multicolinearity problems or loss of degrees of freedom. The variables listed in this table are the 'independent right-hand-side' or ' $X$ ' variables in our spatial models. 
Table 1: New HEV Registrations and HEV Adoption Penetration Reported by Ohio BMV Between 2001 and 2012

\begin{tabular}{llllllllll}
\hline Year & $\begin{array}{l}\text { Ford } \\
\text { Escape }\end{array}$ & $\begin{array}{l}\text { Honda } \\
\text { Civic }\end{array}$ & $\begin{array}{l}\text { Honda } \\
\text { Insight }\end{array}$ & $\begin{array}{l}\text { Lexus } \\
\text { CT200H }\end{array}$ & $\begin{array}{l}\text { Lexus } \\
\text { HS250H }\end{array}$ & $\begin{array}{l}\text { Toyota } \\
\text { Prius }\end{array}$ & $\begin{array}{l}\text { Toyota } \\
\text { Camry }\end{array}$ & $\begin{array}{l}\text { Toyota } \\
\text { Highlander }\end{array}$ & $\begin{array}{l}\text { HEVs/1000 } \\
\text { Vehicles }\end{array}$ \\
\hline 2001 & 0 & 0 & 136 & 0 & 0 & 392 & 0 & 0 & 0.0007667 \\
2002 & 0 & 0 & 100 & 0 & 0 & 626 & 1 & 0 & 0.0009301 \\
2003 & 0 & 1041 & 54 & 0 & 0 & 351 & 0 & 0 & 0.0019820 \\
2004 & 0 & 609 & 25 & 0 & 0 & 1195 & 0 & 0 & 0.0026744 \\
2005 & 295 & 693 & 27 & 0 & 0 & 2962 & 0 & 0 & 0.0059316 \\
2006 & 505 & 1082 & 31 & 0 & 0 & 2211 & 0 & 1019 & 0.0081898 \\
2007 & 341 & 975 & 0 & 0 & 0 & 3838 & 1738 & 518 & 0.0127499 \\
2008 & 619 & 1139 & 0 & 0 & 0 & 4572 & 739 & 203 & 0.0141351 \\
2009 & 373 & 828 & 0 & 0 & 0 & 2262 & 1299 & 85 & 0.0241366 \\
2010 & 221 & 223 & 1678 & 0 & 234 & 5491 & 261 & 159 & 0.0241366 \\
2011 & 170 & 61 & 481 & 187 & 34 & 2792 & 281 & 50 & 0.0134395 \\
2012 & 99 & 310 & 213 & 309 & 4 & 3667 & 1148 & 128 & 0.0133436 \\
\hline Total & 2623 & 6651 & 2745 & 496 & 272 & 30359 & 5186 & 2162 & 0.0042815 \\
\hline
\end{tabular}

Table 2: Selected Demographic and Socioeconomic Variables

\begin{tabular}{ll}
\hline Number & Variable Name \\
\hline Population & Size \\
1 & Number of households in thousands \\
2 & Population in thousands \\
Commute & Method \\
3 & Number of people who drive alone in thousands \\
4 & Number of people who carpool in thousands \\
5 & Number of people who use public transportation in thousands \\
Education & \\
6 & Number of people aged 18 or higher with high school diploma or higher in thousands \\
7 & Number of people aged 18 or higher with bachelor's degree or higher in thousands \\
Vehicle & Ownership \\
8 & Number of households with 0 vehicles in thousands \\
9 & Number of households with 1 vehicle in thousands \\
10 & Number of households with $\geq 2$ vehicles in thousands \\
Income & Median household income in thousand dollars \\
11 & Mean household income in thousand dollars \\
12 &
\end{tabular}

\subsection{Spatial Weighting Matrix}

All three models use the same first-order contiguity matrix to represent the spatial relationships between census tracts. Moreover, we assume $W_{1}=W_{2}$ in the GWR model. A first-order contiguity matrix has zero/one entries, with a one indicating that two census tracts are spatially connected to one another. We consider two census tracts to be spatially connected if a vertex or any portion of a border of one touches a vertex or any portion of a border of the other. Although a first-order contiguity matrix only directly models spatial relationships between adjacent census tracts, it allows indirect relationships between non-adjacent tracts. For instance, if tracts 1 and 2 are adjacent to one another, tract 2 is adjacent to 3 , but tract 1 is not adjacent to 3 , there would only be direct spatial effects between tracts 1 and 2 and tracts 2 and 3 . However, tract 1 indirectly influences tract 3 through its direct effect on tract 2 . 
There is an important limitation arising from our use of census tracts as the spatial units in our analysis. By definition, census tracts only capture the effects of geographic neighbors on HEV adoption. As such, our analysis neglects the effects of 'social neighbors.' For instance, technology experience by a friend or family member, who is not a geographic neighbor, could affect technology adoption. Axsen and Kurani (2011) study the effects of social networks and demonstrate that they affect individuals' perceptions of PEVs. These perception effects could affect subsequent PEV adoption.

\section{Results}

\subsection{Testing for Spatial Autocorrelation}

We use both Moran's $I$ and the Lagrange multiplier error test to check for spatial autocorrelation in the adoption penetration data and errors, respectively. Table 3 summarizes the results of the two tests, showing that both null hypotheses (of no spatial autocorrelation in the adoption penetration and errors) are strongly rejected, because both statistics have $p$-values of 0.0000 . These results suggest that a spatial regression model is more appropriate to capture HEV adoption and provide consistent parameter estimates. Excluding spatial structure from the model can yield biased or inconsistent parameter estimates.

Table 3: Moran's I and Lagrange Multiplier Error Test Results

\begin{tabular}{lll}
\hline & Moran's $I$ & $L M_{e}$ \\
\hline Test Statistic & 6.0172 & 17.6110 \\
$p$-value & 0.0000 & 0.0000 \\
\hline
\end{tabular}

\subsection{Model Estimates}

Table 4 summarizes the maximum-likelihood coefficient estimates for the SAR, SE, and GWR models. The table also provides $t$-statistics and indicates which coefficients are significant at the $5 \%$ and $1 \%$ confidence levels. As Moran's $I$ and the Lagrange multiplier error tests suggest, all three of the models show the existence of statistically significant spatial autocorrelation in the data. The SAR and SE models indicate the existence of autocorrelation in the adoption penetrations and errors, respectively, whereas the GWR model indicates autocorrelation in the adoption penetrations only.

Following the discussion in Section 3.3, we see that the log-likelihood values, AIC, and SBC very slightly favor the SAR model over the other three (smaller AIC and SBC values indicate a better relative model fit to the data). However, AIC, SBC, and the log-likelihood values all make it clear that there is little difference between the three models in terms of goodness of fit, as the values are virtually indistinguishable. It is only at multiple decimal points that differences arise. Alternatively, we can attempt to distinguish between the three models by examining the results of the GWR model in the context of the two restricted models (the other two models can be thought of as restricted versions of GWR, because they only capture one of the two spatial effects captured by the GWR). The only model in which $\lambda$ is statistically significant is the SE model, in which it is the only spatial effect modeled. When both $\lambda$ and $\rho$ are included in the GWR model, only $\rho$ is statistically significant. This reinforces the notion that the SAR model is most appropriate, insomuch as in the presence of $\rho, \lambda$ adds no explanatory power.

The three models all find that there is a statistically significant negative relationship between the percentage of the population holding Bachelor's degree or higher and HEV penetration. The SAR model estimates that for every additional one thousand residents with a bachelor's degree in a census tract, the proportion of HEVs registered declines by $0.64 \%$. This result runs counter to standard intuition that better educated people tend to favor new technology adoption. Interestingly, we find the opposite result when analyzing a time-lagged model in Section 5.3 ( $c f$. Table 6). This finding suggests that the effect of education is sensitive to the model structure and that there may be temporal dynamics in HEV adoption that confound the effect of education if time lags are not modeled. 
Table 4: Maximum Likelihood Estimators for SAR, SE, and GWR Models. $t$-statistics are in parentheses. Coefficients with * and ${ }^{* *}$ indicate significance at the $5 \%$ and $1 \%$ confidence levels, respectively.

\begin{tabular}{|c|c|c|c|}
\hline Variable & SAR & $\mathrm{SE}$ & GWR \\
\hline Constant & $\begin{array}{l}2.7218^{* *} \\
(4.21)\end{array}$ & $\begin{array}{l}3.3900^{* *} \\
(5.22)\end{array}$ & $\begin{array}{l}2.5041^{* *} \\
(3.97)\end{array}$ \\
\hline \# of Households & $\begin{array}{l}2.2746 \\
(1.49)\end{array}$ & $\begin{array}{l}2.1692 \\
(1.42)\end{array}$ & $\begin{array}{l}2.2188 \\
(1.46)\end{array}$ \\
\hline Population & $\begin{array}{l}-0.0090 \\
(-0.03)\end{array}$ & $\begin{array}{l}0.0038 \\
(0.01)\end{array}$ & $\begin{array}{l}-0.0126 \\
(-0.04)\end{array}$ \\
\hline Drive Alone & $\begin{array}{l}-0.8732 \\
(-1.26)\end{array}$ & $\begin{array}{l}-0.8118 \\
(-1.15)\end{array}$ & $\begin{array}{l}-0.8503 \\
(-1.23)\end{array}$ \\
\hline Carpool & $\begin{array}{l}2.0531 \\
(1.17)\end{array}$ & $\begin{array}{l}2.0939 \\
(1.18)\end{array}$ & $\begin{array}{l}2.0491 \\
(1.17)\end{array}$ \\
\hline Public Transportation & $\begin{array}{l}1.3355 \\
(0.43)\end{array}$ & $\begin{array}{l}1.4215 \\
(0.45)\end{array}$ & $\begin{array}{l}1.4112 \\
(0.46)\end{array}$ \\
\hline High School Diploma or Higher & $\begin{array}{l}0.1587 \\
(0.47)\end{array}$ & $\begin{array}{l}0.1566 \\
(0.46)\end{array}$ & $\begin{array}{l}0.1575 \\
(0.46)\end{array}$ \\
\hline Bachelor's Degree or Higher & $\begin{array}{l}-0.6455^{* *} \\
(-2.84)\end{array}$ & $\begin{array}{l}-0.6793^{* *} \\
(-2.92)\end{array}$ & $\begin{array}{l}-0.6350^{* *} \\
(-2.81)\end{array}$ \\
\hline 0 Vehicles & $\begin{array}{l}-2.2331 \\
(-1.06)\end{array}$ & $\begin{array}{l}-2.2673 \\
(-1.06)\end{array}$ & $\begin{array}{l}-2.1769 \\
(-1.04)\end{array}$ \\
\hline 1 Vehicle & $\begin{array}{l}-2.4758^{*} \\
(-2.10)\end{array}$ & $\begin{array}{l}-2.4516^{*} \\
(-2.03)\end{array}$ & $\begin{array}{l}-2.4190^{*} \\
(-2.06)\end{array}$ \\
\hline 2 Vehicles & $\begin{array}{l}-1.0413 \\
(-0.61)\end{array}$ & $\begin{array}{l}-1.0125 \\
(-0.59)\end{array}$ & $\begin{array}{l}-1.0144 \\
(-0.59)\end{array}$ \\
\hline Median Income & $\begin{array}{l}-0.0359 \\
(-1.91)\end{array}$ & $\begin{array}{l}-0.0362 \\
(-1.92)\end{array}$ & $\begin{array}{l}-0.0355 \\
(-1.90)\end{array}$ \\
\hline Mean Income & $\begin{array}{l}0.0382^{* *} \\
(2.59)\end{array}$ & $\begin{array}{l}0.0389^{* *} \\
(2.63)\end{array}$ & $\begin{array}{l}0.0381^{* *} \\
(2.59)\end{array}$ \\
\hline$\rho$ & $\begin{array}{l}0.0270^{* *} \\
(5.83)\end{array}$ & & $\begin{array}{l}0.0350^{* *} \\
(384.62)\end{array}$ \\
\hline$\lambda$ & & $\begin{array}{l}0.0280^{* *} \\
(34.79)\end{array}$ & $\begin{array}{l}-0.0040 \\
(-0.54)\end{array}$ \\
\hline Log-Likelihood & -8885.04 & -8886.06 & -8885.45 \\
\hline $\mathrm{AIC}$ & 3.0238 & 3.0241 & 3.0243 \\
\hline $\mathrm{SBC}$ & 3.0289 & 3.0293 & 3.0298 \\
\hline
\end{tabular}

The other three statistically significant coefficients are more sensible. The number of households with one vehicle have a statistically significant negative effect on vehicle adoption, showing that single-vehicle households are less likely to adopt HEVs. This is in agreement with the conventional wisdom that HEVs are often used as commuter vehicles, because the higher purchase price must be recovered through fuel cost savings accrued over many miles. Commuter vehicles tend to have driving patterns that are more conducive to such cost recovery. Flamm (2009) shows that the number of vehicles owned by a household and the need to use a vehicle for commuting are statistically significantly positively correlated. Another possible explanation of the effect of owning multiple vehicles is the number of persons in the household. A household with a single vehicle may consist of fewer persons, resulting in fewer vehicle trips and miles being driven. Conversely, a household with multiple vehicles is more likely to consist of multiple persons, which could result in more vehicle miles being driven and further opportunity to recover the higher vehicle purchase price through fuel savings. The $\rho$ parameter having a positive value further indicates that higher HEV 
adoption penetrations in an adjacent census tract has a positive effect on HEV adoption penetration within a given census tract.

\subsection{Time-Lagged Results}

To further understand the dynamics of HEV adoption, we examine the effects of time-lagged HEV adoption on subsequent adoption. More specifically, we use the three time-lagged model structures introduced in Section 3.4. We define the HEV adoption penetration within each census tract between the years 2009 and 2012 as the dependent variable (i.e., as the $y$ variable). We then let the HEV adoption penetration within each census tract between the years 2001 and 2008 be the time-lagged variable (i.e., the $\dot{y}$ variable). The explanatory variables in the $X$ matrix remain the same as in the standard model (these are listed in Table 2) and we use the same first-order contiguity matrix as before with $W_{1}=W_{2}$.

Table 5 summarizes the results of Moran's $I$ and the Lagrange multiplier error test with the timelagged model structure. As before, these tests strongly reject the null hypotheses of no autocorrelation in the adoption penetrations and errors, respectively. Table 6 summarizes the maximum-likelihood coefficient estimates for the time-lagged SAR, SE, and GWR models. The AIC, SBC, Moran's I, Lagrange multiplier error test, and $\rho$ and $\lambda$ being significant in all three model structures, suggest that the GWR model is the most appropriate to fully capture the spatial autocorrelation in the data.

Table 5: Moran's I and Lagrange Multiplier Error Test Results for Time-Lagged Model

\begin{tabular}{lll}
\hline & Moran's $I$ & $L M_{e}$ \\
\hline Test Statistic & -3.9910 & 126.0622 \\
$p$-value & 0.0001 & 0.0000 \\
\hline
\end{tabular}

The coefficient on the lagged HEV adoption is significant and has a positive sign in all three models, indicating that HEV purchases in an adjacent census tract between 2001 and 2008 had a positive influence on HEV adoption between 2009 and 2012. This comports with the broader hypothesis that familiarity with HEVs, whether through space, as in the earlier models, or time, has an overall positive effect on further adoption. The other coefficients align with the findings in Table 4. In the time-lagged results, however, bachelor's degree attainment is no longer negatively related to adoption, but high school diploma attainment is. Contrasting this result with the estimates of the base model (without a time lag) suggests that the HEV adoption data may have important time dynamics that need to be modeled. This is because the effects of education are counter-intuitive and run contrary to other studies of technology adoption when time dynamics are not modeled. Including time dynamics gives the expected impacts of education. Population is also significant, indicating that those living in more populous tracts (which are mostly urban) are more likely to own HEVs.

\subsection{Marginal Effect Results}

We can finally examine the impacts of HEV adoption in one census tract on adoption across the study area (i.e., on that and other census tracts). We do this by applying the methodology detailed in Section 3.5 to the model estimates described in Section 5.2. Table 7 summarizes the results of this analysis in the hypothetical case that the adoption rate of HEVs in census tract 1 increases from 4.20103 per thousand registered vehicles to 5.20103 per thousand registered vehicles. The column labeled ' $y^{0}$ ' shows the starting HEV adoption rates in the 2943 census tracts before the marginal-effect analysis is conducted. Thus, these values represent the actual HEV adoption rates determined from the Ohio BMV data. Next, the column labeled ' $y^{\Delta}$ ' shows the HEV adoption rates when the perturbation corresponding to Equation (23) is applied (i.e., the adoption rate in census tract 1 is increased.

The final column shows the effect of the increased HEV adoption rate in census tract 1 on the HEV adoption rates of all of the census tracts. These values are computed iteratively using Equations (25) and (26). A total of five iterations is needed to arrive at the final set of stable HEV adoption rates. We 
Table 6: Maximum Likelihood Estimators for Time-Lagged SAR, SE, and GWR Models. $t$-statistics are in parentheses. Coefficients with ${ }^{*}$ and ${ }^{* *}$ indicate significance at the $5 \%$ and $1 \%$ confidence levels, respectively.

\begin{tabular}{|c|c|c|c|}
\hline Variable & SAR & $\mathrm{SE}$ & GWR \\
\hline Constant & $\begin{array}{l}1.4787^{* *} \\
(3.33)\end{array}$ & $\begin{array}{l}2.5422^{* *} \\
(5.16)\end{array}$ & $\begin{array}{l}1.9914^{* *} \\
(4.26)\end{array}$ \\
\hline \# of Households & $\begin{array}{l}1.6139 \\
(1.58)\end{array}$ & $\begin{array}{l}2.0496^{*} \\
(1.85)\end{array}$ & $\begin{array}{l}1.8755 \\
(1.76)\end{array}$ \\
\hline Population & $\begin{array}{l}0.9475^{* *} \\
(4.12)\end{array}$ & $\begin{array}{l}1.0255^{* *} \\
(4.15)\end{array}$ & $\begin{array}{l}0.9814^{* *} \\
(4.08)\end{array}$ \\
\hline Drive Alone & $\begin{array}{l}-0.6417 \\
(-1.38)\end{array}$ & $\begin{array}{l}-0.7355 \\
(-1.45)\end{array}$ & $\begin{array}{l}-0.6845 \\
(-1.40)\end{array}$ \\
\hline Carpool & $\begin{array}{l}-0.5073 \\
(-0.43)\end{array}$ & $\begin{array}{l}-0.3803 \\
(-0.30)\end{array}$ & $\begin{array}{l}-0.3843 \\
(-0.31)\end{array}$ \\
\hline Public Transportation & $\begin{array}{l}1.1431 \\
(0.55)\end{array}$ & $\begin{array}{l}1.9097 \\
(0.82)\end{array}$ & $\begin{array}{l}1.4885 \\
(0.67)\end{array}$ \\
\hline High School Diploma or Higher & $\begin{array}{l}-0.8455^{\text {** }} \\
(-3.71)\end{array}$ & $\begin{array}{l}-0.9184^{* *} \\
(-3.76)\end{array}$ & $\begin{array}{l}-0.8791^{* *} \\
(-3.70)\end{array}$ \\
\hline Bachelor's Degree or Higher & $\begin{array}{l}0.0799 \\
(0.53)\end{array}$ & $\begin{array}{l}0.0419 \\
(0.25)\end{array}$ & $\begin{array}{l}0.0525 \\
(0.33)\end{array}$ \\
\hline 0 Vehicles & $\begin{array}{l}-0.7327 \\
(-0.52)\end{array}$ & $\begin{array}{l}-1.0954 \\
(-0.72)\end{array}$ & $\begin{array}{l}-0.9694 \\
(-0.66)\end{array}$ \\
\hline 1 Vehicle & $\begin{array}{l}-1.2508 \\
(-1.58)\end{array}$ & $\begin{array}{l}-1.7575^{* *} \\
(-2.01)\end{array}$ & $\begin{array}{l}-1.5667 \\
(-1.88)\end{array}$ \\
\hline 2 Vehicles & $\begin{array}{l}-0.9482 \\
(-0.83)\end{array}$ & $\begin{array}{l}-1.3725 \\
(-1.12)\end{array}$ & $\begin{array}{l}-1.2234 \\
(-1.03)\end{array}$ \\
\hline Median Income & $\begin{array}{l}-0.0144 \\
(-1.14)\end{array}$ & $\begin{array}{l}-0.0195 \\
(-1.47)\end{array}$ & $\begin{array}{l}-0.0175 \\
(-1.34)\end{array}$ \\
\hline Mean Income & $\begin{array}{l}0.0015 \\
(0.15)\end{array}$ & $\begin{array}{l}0.0028 \\
(0.27)\end{array}$ & $\begin{array}{l}0.0027 \\
(0.26)\end{array}$ \\
\hline 2001-2008 HEV Adoption Penetration & $\begin{array}{l}0.0185^{* *} \\
(8.06)\end{array}$ & $\begin{array}{l}0.0206^{* *} \\
(6.33)\end{array}$ & $\begin{array}{l}0.0167^{* *} \\
(6.41)\end{array}$ \\
\hline$\rho$ & $\begin{array}{l}0.0500^{* *} \\
(13.17)\end{array}$ & & $\begin{array}{l}0.0350^{* *} \\
(432.98)\end{array}$ \\
\hline$\lambda$ & & $\begin{array}{l}0.0550^{* *} \\
(10.90)\end{array}$ & $\begin{array}{l}0.0350^{* *} \\
(433.97)\end{array}$ \\
\hline Log-Likelihood & -7720.82 & -7727.75 & -7708.22 \\
\hline $\mathrm{AIC}$ & 2.6282 & 2.6306 & 2.6243 \\
\hline $\mathrm{SBC}$ & 2.6333 & 2.6375 & 2.6298 \\
\hline
\end{tabular}

can see that increased HEV adoption in census tract 1 has mixed spatial effects. Some census tracts, such as 1, 2, 5, 7, and 2943 (among the ones shown), have increased HEV adoption. The others see decreased $\mathrm{HEV}$ adoption. Interestingly, census tract 1 sees increased HEV adoption, however the net HEV adoption rate increases by less than 1 (which is the original perturbation applied to $y^{\Delta}$ ). This means that increasing $\mathrm{HEV}$ adoption in census tract 1 results in a spatially-driven negative feedback effect. To understand the effect intuitively, the increased HEV adoption in census tract 1 has effects on HEV adoption in other census tracts. However, these changes in HEV adoption in the other census tracts affect HEV adoption in census tract 1 , and in net act to hamper HEV adoption in census tract 1 .

In total, one could compute a $2943 \times 2943$ matrix showing the marginal effects of HEV adoption in one census tract on adoption in all of the other census tracts. The differences in the values reported in the $y^{5}$ 
Table 7: Marginal Effect of Increased HEV Adoption in Census Tract 1

\begin{tabular}{llll}
\hline Census Tract & $y^{0}$ & $y^{\Delta}$ & $y^{5}$ \\
\hline 1 & 4.20103 & 5.20103 & 4.91045 \\
2 & 4.88051 & 4.88051 & 5.17487 \\
3 & 4.16361 & 4.16361 & 3.88646 \\
4 & 3.40657 & 3.40657 & 2.96158 \\
5 & 2.99238 & 2.99238 & 3.44134 \\
6 & 1.70940 & 1.70940 & 1.20369 \\
7 & 8.49721 & 8.49721 & 8.59076 \\
$\vdots$ & $\vdots$ & $\vdots$ & $\vdots$ \\
2943 & 6.28667 & 6.28667 & 7.17936 \\
\hline
\end{tabular}

and $y^{0}$ columns of Table 7 provide one column of such a matrix, because they represent the effect of HEV adoption in census tract 1 on adoption across all of the census tracts. We do not compute and present a full $2943 \times 2943$ matrix because doing so is computationally time consuming and the values would be difficult to present in tabular or graphical form.

\section{Conclusions}

In this paper we analyze spatial effects on $\mathrm{HEV}$ adoption using census tract-level demographic data and vehicle registrations reported for the state of Ohio. HEV adoption can be used as a proxy for future PEV adoption, insomuch as similar dynamics involving early adopters and a subsequent wave of broader adoption is seen with many new technologies. Nevertheless, it is important to stress that are likely to be some differences between HEV and PEV adopters. PEV adopters (especially early on, before widespread availability of charging infrastructure) may need to consider range-limitations imposed by an all-electric vehicle. Properly capturing the clustering of HEVs and potential clustering of future PEVs is important for charging and electric power system infrastucture planning. Moran's $I$, the Lagrange multiplier error test, and the significance of the spatial autocorrelation parameters in the SAR, SE, and GWR models demonstrate the importance of including a spatial structure in HEV adoption analysis. Omitting this structure can result in biased or inconsistent parameter estimates. The spatial effect is shown to be positive, meaning that there is indeed a neighbor effect-people tend to adopt HEVs when their geographic neighbors adopt HEVs. However, as our marginal analysis conducted in Section 5.4 shows, HEV adoption in some census tracts can have a negative effect on adoption elsewhere. We also conduct a time-lagged analysis, which shows that previous HEV adoption has the same overall positive effect on subsequent HEV adoption. Again, this follows the conventional wisdom that people tend to adopt HEVs when their neighbors have previously done so.

We also demonstrate the use of the spatial models to estimate the marginal effects of HEV adoption in one spatial unit on HEV adoption everywhere. Doing a complete analysis of this type requires an iterative technique to capture all of the spatial spillover effects, which in our case study would result in generating a $2943 \times 2943$ matrix summarizing the marginal effects. Such an analysis can be used to simulate the rate of HEV adoption across a spatial domain and can be useful for many vehicle-related analyses. This can include planning the location of PEV-charging infrastructure and upgrades to electricity-distribution infrastructure for PEV charging (using HEV adoption as a proxy for future PEV adoption). Another important use of a marginal analysis is to study how to target and focus incentive and similar programs for early adoption of HEVs and PEVs. Our results show that early adoption has an overall positive effect on subsequent technology adoption. This is shown by lagged HEV adoption having a statistically significant positive value in all three of the time-lagged SAR, SE, and GWR models ( $c f$. Table 6). Policymakers with the goal of encouraging widespread use of HEVs and PEVs can determine how to tailor incentive programs to increase 
the adoption of HEVs and PEVs by early adopters. Moreover, incentive programs can be tailored toward early adopters that have the greatest spatial 'spillover' effect on subsequent technology adoption. This spillover effect can be estimated or simulated using the marginal analysis discussed in Section 3.5.

The analysis conducted here focuses on HEV adoption in the state of Ohio. It is important to stress, however, that the case study conducted here can be generalized to other study regions, so long as the input data used are available. Many jurisdictions maintain socioeconomic, demographic, and vehicle-registration data, which can be used to repeat our analysis elsewhere. There are a number of important insights from this analysis that have implications for future transportation use. One is that our work demonstrates how spatial econometric models can be applied to discern spatial relationships in the adoption or new transportation technologies. Second, the patterns of HEV adoption can be used to proactively plan PEVrelated infrastructure in anticipation of where PEVs may be adopted. As PEV adoption takes hold, the same types of models can be reestimated using the PEV-adoption data. The model results and coefficient estimates can be used to simulate and predict subsequent PEV adoption.

There are some important limitations to the work presented here, which raise areas of future research. One is that our analysis considers geographic neighbors only in constructing the spatial weighting matrix. This does not capture the effect of social networks and interactions, which could influence HEV adoption. Axsen and Kurani (2011) study the effects of social networks and demonstrate that they affect individuals' perceptions of PEVs. Analogously, HEV adoption decisions by social neighbors, who are not geographic neighbors, may affect an individual's HEV adoption decision. Such an analysis is limited by the difficulty of constructing social networks. Social networking data is increasingly available through social media outlets, however, which may make such an analysis possible. Another area of future research is to focus on a smaller study region (e.g., Central Ohio only), which would allow for including a more granular spatial weighting matrix. Our analysis uses census tracts, which typically have population sizes between 1200 and 8000 people, as the spatial unit. More interesting spatial autocorrelations may appear if this analysis is extended to consider individual households and their immediate geographic neighbors as spatial units. The only limitation of conducting such an analysis is that the size of the dataset increases considerably, which can raise memory and model-estimation difficulties.

\section{Acknowledgments}

The authors thank Armin Sorooshian, the editors, and two anonymous reviewers for helpful discussions and suggestions. This material is based upon work supported by the U.S. Department of Energy under Award Number DE-PI0000012. Financial support for this work was also provided by the SMART@CAR consortium. Any opinions and conclusions expressed in this paper are solely those of the authors.

\section{References}

Anselin, L., 1988. Spatial Econometrics: Methods and Models. Studies in Operational Regional Science. Springer.

Anselin, L., Florax, R., Rey, S. J., 2004. Advances in Spatial Econometrics: Methodology, Tools and Applications. Advances in Spatial Science. Springer-Verlag, Berlin, Germany.

Axsen, J., Kurani, K. S., March 2011. Interpersonal influence in the early plug-in hybrid market: Observing social interactions with an exploratory multi-method approach. Transportation Research Part D: Transport and Environment 16, $150-159$.

Axsen, J., Kurani, K. S., May 2012. Interpersonal Influence within Car Buyers' Social Networks: Applying Five Perspectives to Plug-in Hybrid Vehicle Drivers. Environment and Planning A 44, 1047-1065.

Block, D., Harrison, J., January 2014. Electric Vehicle Sales and Future Projections. Tech. Rep. EVTC-RR-01-14, Electric Vehicle Transportation Center.

Collins, M. M., Mader, G. H., February 1983. The Timing of EV Recharging and Its Effect on Utilities. IEEE Transactions on Vehicular Technology VT-32, 90-97.

Curtin, R., Shrago, Y., Mikkelsen, J., August 2009. Plug-in Hybrid Electric Vehicles, working Paper.

Egbue, O., Long, S., September 2012. Barriers to widespread adoption of electric vehicles: An analysis of consumer attitudes and perceptions. Energy Policy 48, 717-729.

Flamm, B., June 2009. The impacts of environmental knowledge and attitudes on vehicle ownership and use. Transportation Research Part D: Transport and Environment 14, 272-279.

Gallagher, K. S., Muehlegger, E., January 2011. Giving green to get green? Incentives and consumer adoption of hybrid vehicle technology. Journal of Environmental Economics and Management 61, 1-15. 
LeSage, J., Pace, R. K., 2009. Introduction to Spatial Econometrics. Statistics: A Series of Textbooks and Monographs. Chapman \& Hall/CRC, Boca Raton, FL.

Mohseni, P., Stevie, R. G., 26-30 July 2009. Electric vehicles: Holy grail or Fool's gold. In: 2009 Power and Energy Society General Meeting. Institute of Electrical and Electronics Engineers, Calgary, AB, pp. 1-5.

Moran, P. A. P., June 1950. Notes on Continuous Stochastic Phenomena. Biometrika 37, 17-23.

Musti, S., Kockelman, K. M., October 2011. Evolution of the household vehicle fleet: Anticipating fleet composition, PHEV adoption and GHG emissions in Austin, Texas. Transportation Research Part A: Policy and Practice 45, 707-720.

Paelinck, J. H. P., Klaassen, L. H., 1979. Spatial Econometrics (Studies in spatial analysis). Saxon House.

Plötz, P., Schneider, U., Globisch, J., Dütschke, E., September 2014. Who will buy electric vehicles? Identifying early adopters in Germany. Transportation Research Part A: Policy and Practice 67, 96-109.

Rezvani, Z., Jansson, J., Bodin, J., January 2015. Advances in consumer electric vehicle adoption research: A review and research agenda. Transportation Research Part D: Transport and Environment 34, 122-136.

Sioshansi, R., Denholm, P., February 2009. Emissions Impacts and Benefits of Plug-in Hybrid Electric Vehicles and Vehicle to Grid Services. Environmental Science and Technology 43, 1199-1204.

Sioshansi, R., Denholm, P., 2010. The value of plug-in hybrid electric vehicles as grid resources. The Energy Journal 31 , 1-23.

Tuttle, D. P., Baldick, R., August-September 2015. Technological, Market and Policy Drivers of Emerging Trends in the Diffusion of Plug-in Electric Vehicles in the U.S. The Electricity Journal 28, 29-34.

Ward, M. D., Gleditsch, K. S., 2008. Spatial Regression Models. Quantitative Applications in the Social Sciences. Sage Publications, Inc., Thousand Oaks, CA. 